\title{
Community Based Network Reconstruction for an Evolutionary Algorithm Framework
}

\author{
Dr. V. Suma \\ Professor, \\ Department of Information Science \& Engineering, \\ Dayananda Sagar College of Engineering, \\ Bangalore, India.
}

\begin{abstract}
Inferring complex and non-linear dynamic system using the data that is available plays an important role in many areas of work such as physical, social, biological and computer sciences. In order to address these issues, network structure using a number of evolutionary algorithms has been proposed. However, the important criteria like the community structure have been ignored while developing these methodologies. Accordingly, this proposed work is focused on developing a multi-objective network reconstruction based on community structure in order to improve the network construction using ES by boosting their reconstruction performance. This framework that is used to further improve their performance is known as the community-based framework. It is based on multi-objective metaheuristic algorithm that is based on population and can be used as the base optimizer. The original decision space of the community structure is divided using the proposed work. From the solution obtained, an improved solution using reduced decision space is implemented using the multi-objective evolutionary algorithm (MOEA). A test suite is also designed to verify the performance of community based network reconstruction with respect to the complex network issue. In the proposed reconstruction methodology based on community criteria, the MOEAs are incorporated and are used to bind the original version. A noticeable improvement is seen in the experimental results based on the proposed work on 30 reconstruction issues.
\end{abstract}

Keywords: Community service, evolutionary algorithm, muti-objective optimization, complex network reconstruction 
Journal of Artificial Intelligence and Capsule Networks (2021)

Vol.03/ No.01

Pages: 53-61

http://irojournals.com/aicn/

DOI: https://doi.org/10.36548/jaicn.2021.1.005

\section{Introduction}

Analysis on the issues of synchronizing and controlling the complex and nonlinear dynamical system is impressive and has caught the attention of many researchers in the various fields of study such as social, biological, computer, physical and engineering sciences. To analyze the complex dynamics, the complex networks play an important role and will also be used to control the collective dynamics. Every part of key factor involved in a complex system will be used as a node or a concept using the complex network. However, as far as control of the collective dynamics is concerned, it will be a difficult task to control due to the nodal dynamics in the complex system and the network structure which remain unknown [1]. Only a handful of observed data is available for use in many cases of complex dynamics. Hence using this data to infer the network structure will be challenging in the engineering and contemporary network science. To overcome this challenge, a large collection of network inference methods have been designed like evolutionary algorithms, random forest, mutual information, regression, etc [2]. To solve complex and non-convex optimization problem, the EA will serve as a powerful tool of optimization. The wide-applications of the EA-based methodologies and their improved performance in tackling reconstruction problems in complex network have been focused in this proposed paper, using EA-based network reconstruction methods [3]. The fuzzy cognitive maps, S-system, observed data and other inference models that is present can be used to infer networks by means of the proposed EAbased network reconstruction [4-5].

Based on the models, the parameters are optimized with the aid of EA. The known data ' $\mathrm{Y}$ ' is used to determine the structure of the network ' $\mathrm{X}$ ' to determine the connection between the individual nodes [6]. Moreover, to recover the network structure, the problem of complex network can be further simplified such that

$$
\min _{x}(h(X, Y), \lambda g(X))
$$

where the value of $\lambda$ should be a positive constant (greater than 0 ). $\mathrm{X}$ denotes the parameters [7] of the network structure or the inference mode. $\mathrm{h}($.) represents the difference between $\mathrm{Y}$ and the inference model output. In eq (1) the difference between the observed data and the output of the dynamic or inference model and $g(X)$ can be utilized to implement a concise network structure. In power networks, gene regulatory networks and social networks that are used as complex networks, a sparse structure is followed. Hence these networks need to be 
Journal of Artificial Intelligence and Capsule Networks (2021)

Vol.03/ No.01

Pages: 53-61

http://irojournals.com/aicn/

DOI: https://doi.org/10.36548/jaicn.2021.1.005

properly inferred to ensure a sparse network structure [8]. Using EA, the non-convex optimization problem can be overcome and eq (1) have proven to be the apt solution.

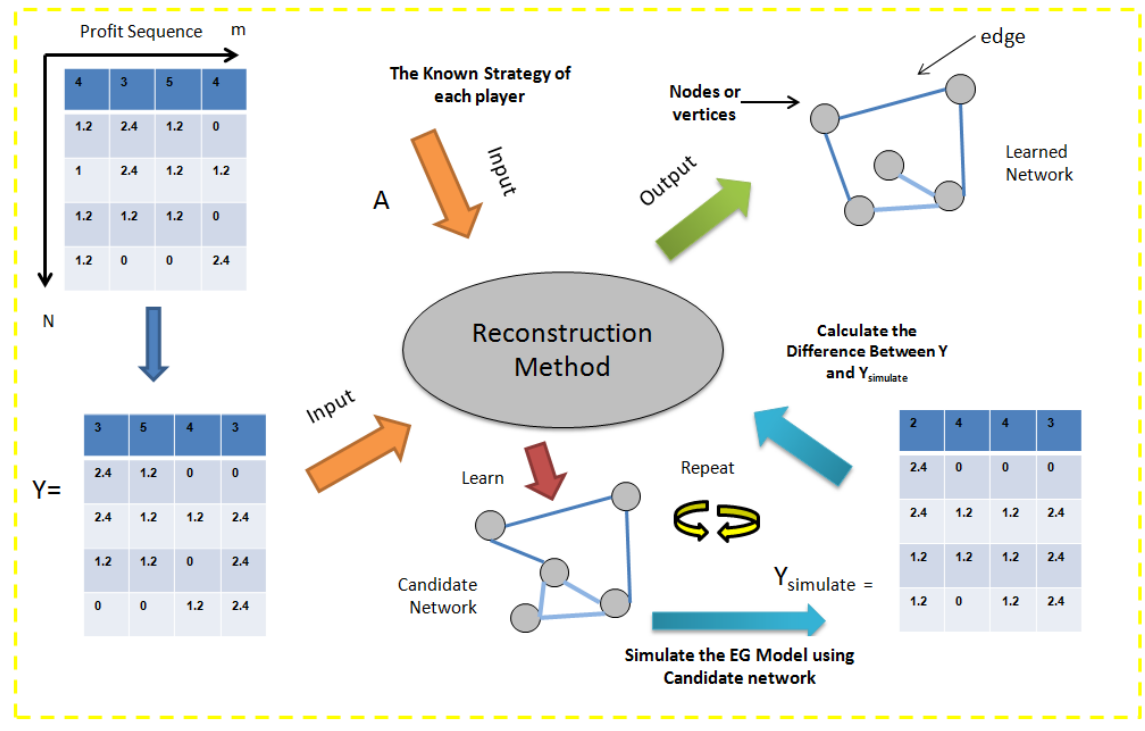

Fig.1.

The value of $\lambda$ will also have a significant impact on the network reconstruction performance that is to be carried out [9]. Hence it is possible to extend the issue in eq (1) to multi-objective problems in order to prevent the choice of $\lambda$ value. A number of EA-based methodologies have been formulated is the recent years, to address this issue [10]. However, it has been observed that these methodologies remain unsatisfactory. On a common note, as the complex system scale begins to elevate in a linear fashion, the complexity of the search space learning algorithm will also increase in an exponential manner [11]. While incorporating in a large-scale complex system, the exact network structure among the nodes is required immediately. To determine this value, a gene regulatory network (GRN) that performs in a large scale requirement will result in a concise description with respect to the genes. Accordingly, the inferred GRN can be used to knock out or control the mutated genes in treating cancer [12]. In order to overcome this limitation imposed by the existing network reconstruction needs to be addressed and this paper develops an optimal algorithm for the same. We have introduced a community-based network reconstruction methodology that can be used instead of the existing reconstruction methods. 
Journal of Artificial Intelligence and Capsule Networks (2021)

Vol.03/ No.01

Pages: 53-61

http://irojournals.com/aicn/

DOI: https://doi.org/10.36548/jaicn.2021.1.005

In general, the social networks are segregated into communities or groups. When a particularly node belongs to the same group, the nodes are linked more densely when compared with those present outside the group. Based on the community sharing structure that is prevalent in the social networks, we have addressed the high-dimensional network reconstruction issue. This issue is first subdivided in many problems of low-dimensions as per the learned community structure. The basic concept behind this process is to segregate the actual problem into smaller and tackle-able sub-problems. In this paper, we have introduced an optimization technique with two stages. Here, the links between or within a community is focused while the entire structure is ignored [13]. This is especially the case when it proves to be an inseparable problem. This will further result in loss or low accuracy in the actual data when it is being decomposed. To overcome this issue, the gap between the decomposed problem and the original problem is bridged using the proposed strategy.

The proposed work can be capsulated into two steps as follows:

1. A network reconstruction based on community is proposed such that it can disseminate the original problem in sub-problems that hold the same property of community structure.

2. To overcome the community structure decomposition issue, a two-stage optimization methodology is introduced. The first stage involves getting the network structure that will be used in the next stage. The second stage involves decomposition of the network reconstruction according to the links between and within the communities. This step is repeated to obtain a higher accuracy.

\section{Proposed Work}

In this proposed work, we have used Resistor network $(\mathrm{RN})$ reconstruction, EG network reconstruction and Fuzzy cognitive map reconstruction problem as examples. When multiple conflicting objectives occur as a part of the optimization problem, it can also be known as the MOPs. This can be formulated mathematically using the formula:

$$
\begin{gathered}
z=\min F(y)=\left(f_{1}(y), f_{2}(y), \ldots, f_{M}(x)\right) \\
y \in \Omega^{D}
\end{gathered}
$$

where $\mathrm{D}$ will represent the number of decision variable and $\mathrm{M}$ will denote conflicting objectives. In the multi-objective problems, there is no single optimal solution which will be suitable for the proposed system. Fig.2 represents a BGLL algorithm where the communities 
Journal of Artificial Intelligence and Capsule Networks (2021)

Vol.03/ No.01

Pages: 53-61

http://irojournals.com/aicn/

DOI: https://doi.org/10.36548/jaicn.2021.1.005

are considered to be $\mathrm{c} 1, \mathrm{c} 2$ and $\mathrm{c} 3$ denotes values from 1 to 12 . Consider a single layer network which has $\mathrm{G}$ as the complex network model such that $\mathrm{G}=(\mathrm{V}, \mathrm{E})$ where $\mathrm{E}$ denotes the edges and $\mathrm{V}$ is the vertices of the nodes. $\mathrm{L}$ will denote the number of edges while $\mathrm{N}$ will denote the number of nodes. Using this, a typical network structure can be formulated as matrix $\mathrm{Y}$ of dimensions $\mathrm{NxN}$

$$
Y=\left[\begin{array}{ccccc}
y_{11} & \ldots & \ldots & \ldots & y_{1 N} \\
\ldots & \ldots & \ldots & \ldots & \ldots \\
y_{N 1} & \ldots & \ldots & \ldots & y_{N N}
\end{array}\right]
$$

Here $y_{i, j} \in\{0,1\}$ which represents the connection between the nodes $\mathrm{j}$ and $\mathrm{i}$ such that it could be any value between 1 and $\mathrm{N}$. One should also remember that a node can be connected to another node, but not to itself. Consider the equation $y_{i, j} \in[-1,1]$. If $y_{i, j}<0$, it will indicate inhibiting effect, while $y_{i, j}>0$ will indicate promoting effect in a FCM. Let $\mathrm{Z}$ be the data observed. Then $\mathrm{h}(\mathrm{Y}, \mathrm{Z})$ will represent the simulation using network reconstruction in the candidate network structure of the inference model. $\mathrm{Y}$ is the network structure that is generated for $\mathrm{Z}$ which can be used as the inference model. The parameters can be minimized as shown below:

$$
\begin{gathered}
Z: \min _{x}(h(Y, Z), g(Y)) \\
\mathrm{Y} \in \Omega^{D}
\end{gathered}
$$

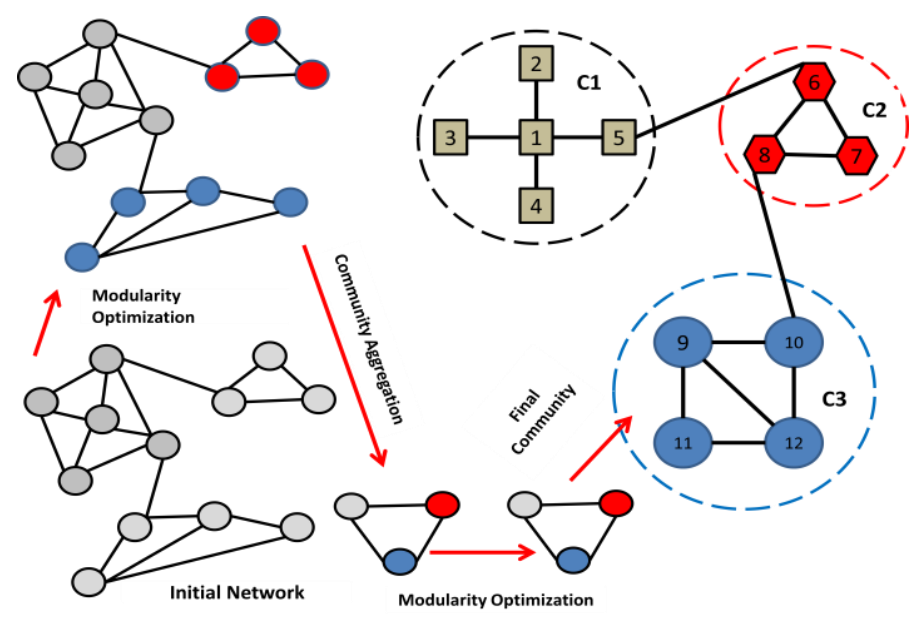

Fig.2.

In the EG model, choice must be made by the agent in selecting cooperation or defection in a particular round. In this proposed work, a payoff matrix for the PDG (prisoner's dilemma games) is used and can be represented as shown below: 
Journal of Artificial Intelligence and Capsule Networks (2021)

Vol.03/ No.01

Pages: 53-61

http://irojournals.com/aicn/

DOI: https://doi.org/10.36548/jaicn.2021.1.005

$$
p=\left(\begin{array}{ll}
1 & 0 \\
1.2 & 0
\end{array}\right)
$$

Here the agents will receive a 1 or 0 if both choose to either defeat or cooperate respectively. On the other hand, if they choose different strategies, they will be rewarded with 0 or 1.2. For every agent, the payment in a particular round can be shown as:

$$
Y_{1}(t)=\sum_{v=1}^{N} y_{u v} S_{u}^{T}(t) P S_{v}(t)
$$

where ' $u$ ' represents the agent in the $\mathrm{t}^{\text {th }}$ round and the strategy of the agent is $S_{u}^{T}(t)$ and T represents transpose. Simulation of data in EG can be done in 3 steps. First the target network is given as the input and every node in the network is considered to be an agent to choose defection or cooperation. In the next step, payoff is calculated and the agent's strategy is continuously updated.

Similarly, the resistor network reconstruction problem will hold a network of resistors that will be used to transfer information in the form of current. Here the reconstruction problem with multi-objectives can be represented as eq (8):

$$
h(X, Y)=\sum_{u=1}^{N}\left\|R_{u} Y_{u}-Z_{u}\right\|_{2}^{2}
$$

Numerical simulation of the RN can be done using 3 steps similar to that of the EG methodology. A random value is generated from a weighted value of $[0,20]$, In the next step, the voltage of the node through which current flows is calculated and then the electrical current that is also flowing through is calculated. This step is repeated for iterative $\mathrm{m}$ time and the values obtained are recorded.

\section{Results and Discussion}

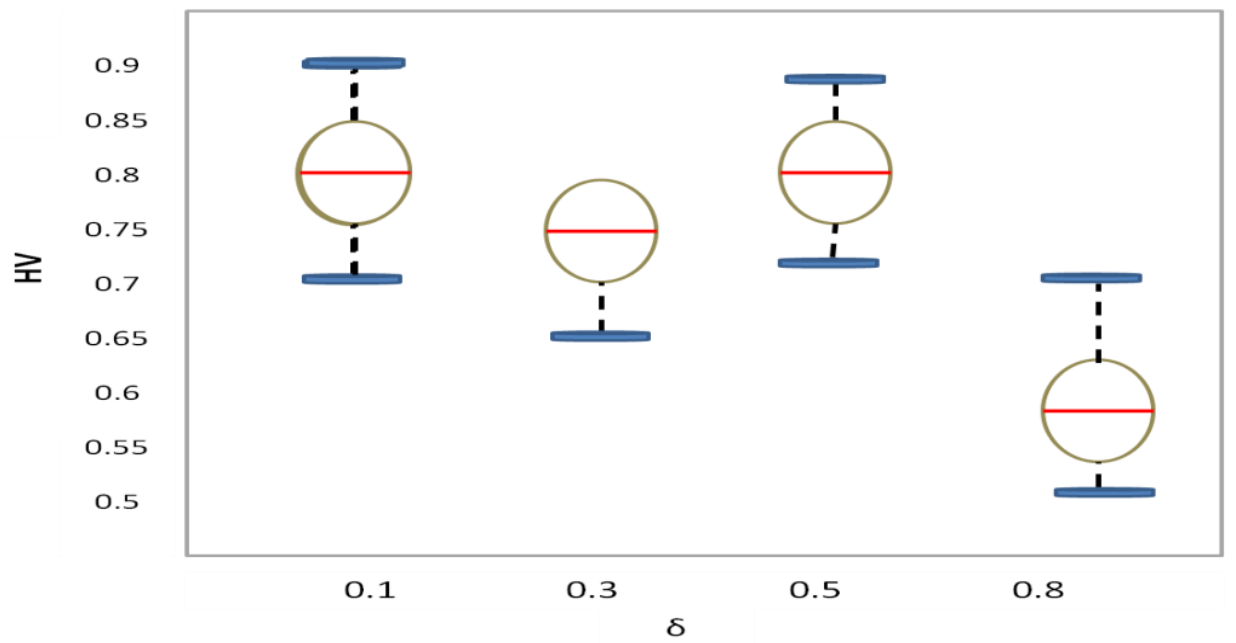

Fig.3. HV for varying sets of $\delta$ 
Journal of Artificial Intelligence and Capsule Networks (2021)

Vol.03/ No.01

Pages: 53-61

http://irojournals.com/aicn/

DOI: https://doi.org/10.36548/jaicn.2021.1.005

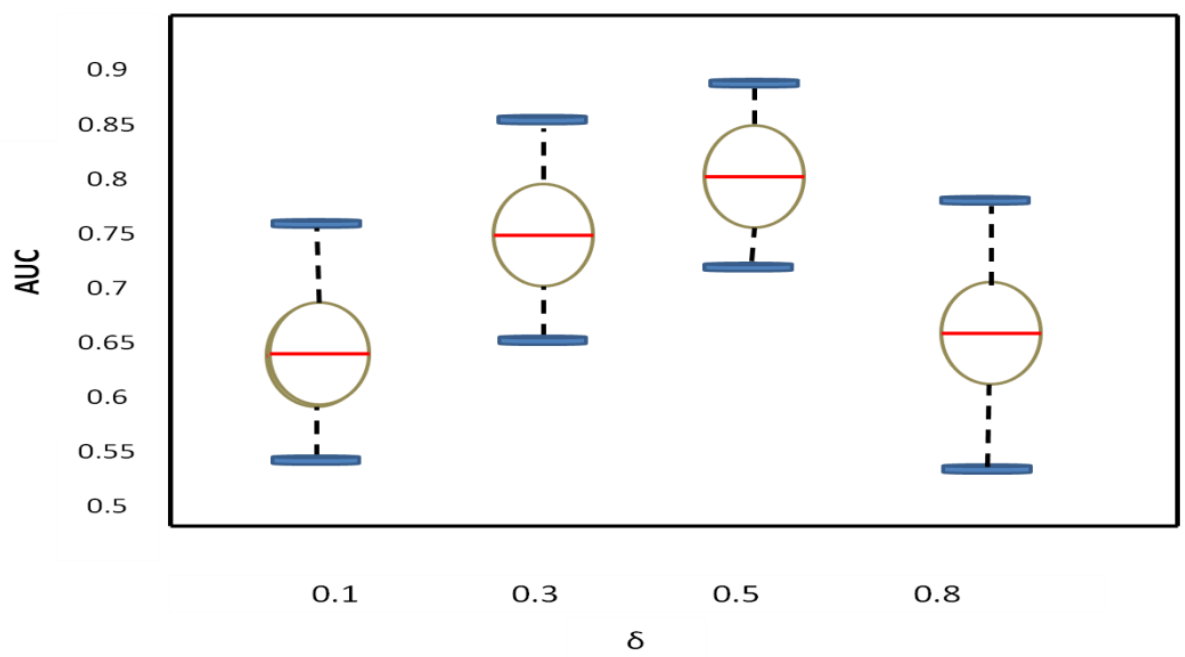

Fig.4. AUC for varying sets of $\delta$

Based on the simulation results, it is observed that for varying values of $\delta$, the corresponding values of $\mathrm{HV}$ and AUC will vary in the range of 0 to 0.8 . As the value of $\delta$ increases, it is seen that in HV, there is a decrease in HV as shown in Fig.3 and in AUC, a rise is observed till 0.5 as shown in Fig.4. However, it is to be noted that as $\delta$ increases, the count of finite errors decreases. This phenomenon is called as normal optimization.

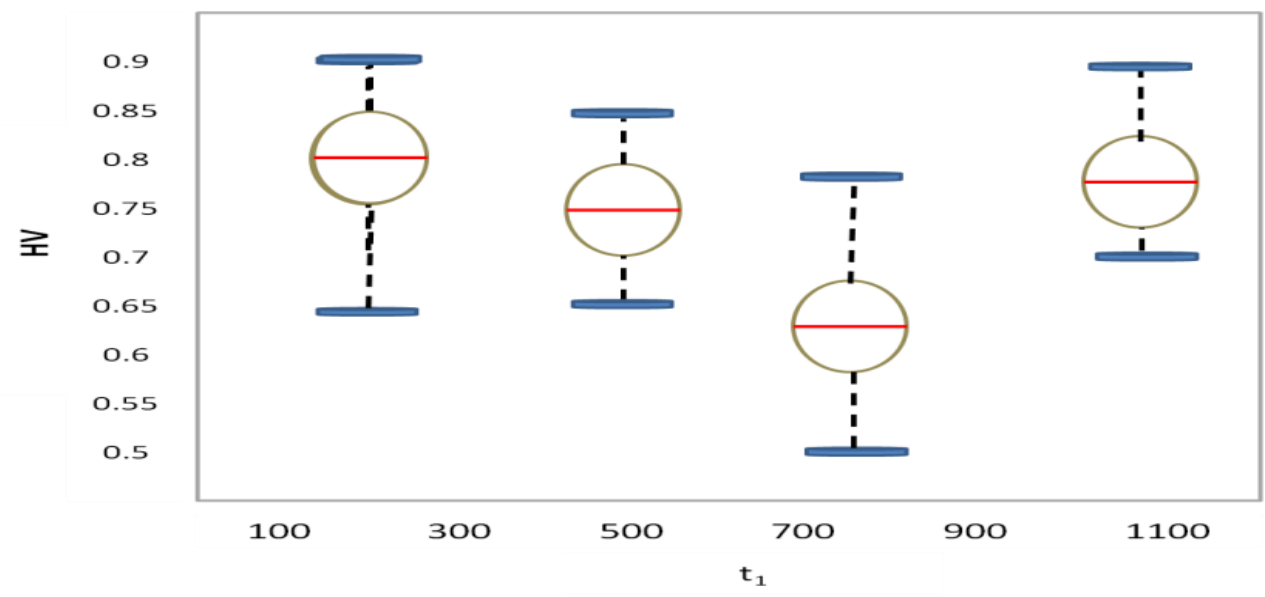

Fig.5. HV for varying sets of $t_{1}$

In Fig.5 and Fig.6, the proposed methodology is executed for varying values of $t_{1}$. It shows that for varying values of $t_{1}$ for values from 100 to 1000 . The varying values will have an impact on HV value. Similarly, for different values of $t_{1}$ for values from 100 to 1000 for AUC values to decompose the issue immediately as the AUC will reach an accurate value. 
Journal of Artificial Intelligence and Capsule Networks (2021)

Vol.03/ No.01

Pages: 53-61

http://irojournals.com/aicn/

DOI: https://doi.org/10.36548/jaicn.2021.1.005

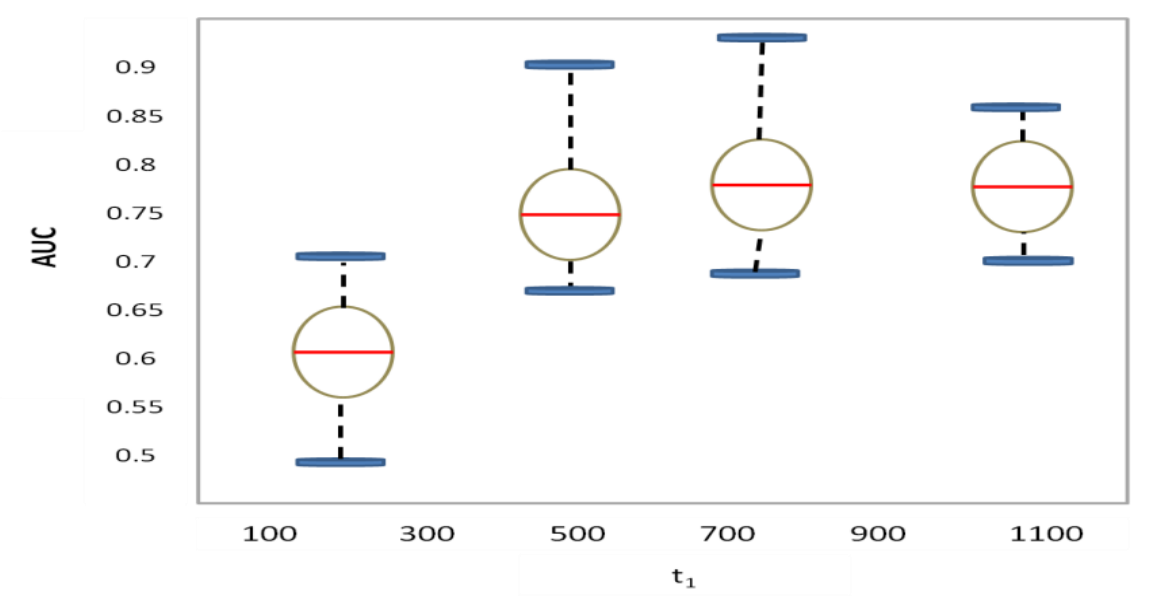

Fig.6. AUC for varying sets of $t_{1}$

\section{Conclusion}

The performance of the community-based network reconstruction is found to be very poor because of the EA. This information of community structure will split the decision for the proposed work. This methodology proves to be apt for the networks and using the proposed work will decompose the issue due to high dimension at a low time complexity. Hence this work will prove to be effective for a wide range of applications. The simulation results are conducted on the proposed work which is compared with previous existing EAs and is found to be extremely effective.

\section{References}

[1] Yao, H., Dai, F., Zhang, S., Zhang, Y., Tian, Q., \& Xu, C. (2019). Dr2-net: Deep residual reconstruction network for image compressive sensing. Neurocomputing, 359, 483-493.

[2] Mi, Z., Luo, Y., \& Tao, W. (2020). SSRNet: scalable 3D surface reconstruction network. In Proceedings of the IEEE/CVF Conference on Computer Vision and Pattern Recognition (pp. 970-979).

[3] Smys, S., Basar, A., \& Wang, H. (2020). Artificial Neural Network Based Power Management for Smart Street Lighting Systems. Journal of Artificial Intelligence, 2(01), 42-52. 
Journal of Artificial Intelligence and Capsule Networks (2021)

Vol.03/ No.01

Pages: 53-61

http://irojournals.com/aicn/

DOI: https://doi.org/10.36548/jaicn.2021.1.005

[4] Sun, L., Fan, Z., Ding, X., Huang, Y., \& Paisley, J. (2019, June). Joint cs-mri reconstruction and segmentation with a unified deep network. In International conference on information processing in medical imaging (pp. 492-504). Springer, Cham.

[5] Zhang, X., Huang, W., Wang, Q., \& Li, X. (2020). SSR-NET: Spatial-Spectral Reconstruction Network for Hyperspectral and Multispectral Image Fusion. IEEE Transactions on Geoscience and Remote Sensing.

[6] Li, B., Yan, Q. R., Wang, Y. F., Yang, Y. B., \& Wang, Y. H. (2020). A binary sampling Res2net reconstruction network for single-pixel imaging. Review of Scientific Instruments, 91(3), 033709.

[7] Wang, Y., Bai, H., Zhao, L., \& Zhao, Y. (2018). Cascaded reconstruction network for compressive image sensing. EURASIP Journal on Image and Video Processing, 2018(1), 1-16.

[8] Vijayakumar, T. (2019). Neural network analysis for tumor investigation and cancer prediction. Journal of Electronics, 1(02), 89-98.

[9] Shirley, Duraisingh Ruth Anita, Deva Aruldhas Janeera, Jeganatha Jeya Padmini, Saleem Mahappuja Asha Banu, and Tharmavel Abirami. "Modelling and Analysis Of Modified Baugh-Wooley Multiplier Using Gate Diffusion Input and Improved Shannon Adder." International Journal of Pure and Applied Mathematics 118, no. 22 (2018): 773-777.

[10] Hu, Z., Xue, H., Zhang, Q., Gao, J., Zhang, N., Zou, S., \& Zheng, H. (2020). DPIRNet: Direct PET image reconstruction based on the Wasserstein generative adversarial network. IEEE Transactions on Radiation and Plasma Medical Sciences, 5(1), 35-43.

[11] Raj, J. S., \& Ananthi, J. V. (2019). Recurrent neural networks and nonlinear prediction in support vector machines. Journal of Soft Computing Paradigm (JSCP), 1(01), 33-40.

[12] Baek, N. R., Cho, S. W., Koo, J. H., Truong, N. Q., \& Park, K. R. (2019). Multimodal camera-based gender recognition using human-body image with two-step reconstruction network. IEEE Access, 7, 104025-104044.

[13] Raj, A., Bresler, Y., \& Li, B. (2020, November). Improving robustness of deeplearning-based image reconstruction. In International Conference on Machine Learning (pp. 7932-7942). PMLR. 